\title{
Commutator Theorems for Fractional Integral Operators on Weighted Morrey Spaces
}

\author{
Zhiheng Wang ${ }^{1}$ and Zengyan $\mathrm{Si}^{2}$ \\ ${ }^{1}$ School of Computer Science and Technique, Henan Polytechnic University, Jiaozuo 454000, China \\ ${ }^{2}$ School of Mathematics and Information Science, Henan Polytechnic University, Jiaozuo 454000, China \\ Correspondence should be addressed to Zengyan Si; zengyan@hpu.edu.cn
}

Received 10 December 2013; Accepted 18 May 2014; Published 1 June 2014

Academic Editor: Guozhen Lu

Copyright (c) 2014 Z. Wang and Z. Si. This is an open access article distributed under the Creative Commons Attribution License, which permits unrestricted use, distribution, and reproduction in any medium, provided the original work is properly cited.

Let $L$ be the infinitesimal generator of an analytic semigroup on $L^{2}\left(\mathbb{R}^{n}\right)$ with Gaussian kernel bounds, and let $L^{-\alpha / 2}$ be the fractional integrals of $L$ for $0<\alpha<n$. For any locally integrable function $b$, the commutators associated with $L^{-\alpha / 2}$ are defined by $\left[b, L^{-\alpha / 2}\right](f)(x)=b(x) L^{-\alpha / 2}(f)(x)-L^{-\alpha / 2}(b f)(x)$. When $b \in \mathrm{BMO}(\omega)$ (weighted BMO space) or $b \in \mathrm{BMO}$, the authors obtain the necessary and sufficient conditions for the boundedness of $\left[b, L^{-\alpha / 2}\right]$ on weighted Morrey spaces, respectively.

\section{Introduction and Main Results}

Morrey [1] introduced the classical Morrey spaces to investigate the local behavior of solutions to second order elliptic partial differential equations. Chiarenza and Frasca [2] established the boundedness of the Hardy-Littlewood maximal operator, the fractional operator, and a singular integral operator on the Morrey spaces. On the other hand, Coifman and Fefferman [3] and Muckenhoupt [4] studied the boundedness of these operators on weighted $L^{p}$ spaces. Motivated by these works, Komori and Shirai [5] introduced the following weighted Morrey space and investigated the boundedness of classical operators in harmonic analysis, that is, the Hardy-Littlewood maximal operator, a CalderónZygmund operator, the fractional integral operator, and so forth.

Let $1 \leq p<\infty$ and $0 \leq k<1$. Then for two weights $\mu$ and $v$, the weighted Morrey space is defined by

$$
L^{p, k}(\mu, \nu)=\left\{f \in L_{\mathrm{loc}}^{p}(\mu):\|f\|_{L^{p, k}(\mu, \nu)}<\infty\right\},
$$

where

$$
\|f\|_{L^{p, k}(\mu, v)}=\sup _{Q}\left(\frac{1}{\nu(Q)^{k}} \int_{Q}|f(x)|^{p} \mu(x) d x\right)^{1 / p}
$$

and the supremum is taken over all balls $Q$ in $\mathbb{R}^{n}$.
If $\mu=\nu$, then we have the classical Morrey space $L^{p, k}(\mu)$ with measure $\mu$. When $k=0$, then $L^{p, k}(\mu, \nu)=L^{p}(\mu)$ is the Lebesgue space with measure $\mu$.

Suppose that $L$ is a linear operator on $L^{2}\left(\mathbb{R}^{n}\right)$ which generates an analytic semigroup $e^{-t L}$ with a kernel $p_{t}(x, y)$ satisfying a Gaussian upper bound, that is,

$$
\left|p_{t}(x, y)\right| \leq \frac{C}{t^{n / 2}} e^{-c\left(|x-y|^{2} / t\right)}
$$

for $x, y \in \mathbb{R}^{n}$ and all $t>0$.

For $0<\alpha<n$, the fractional integral $L^{-\alpha / 2}$ of the operator $L$ is defined by

$$
L^{-\alpha / 2} f(x)=\frac{1}{\Gamma(\alpha / 2)} \int_{0}^{\infty} e^{-t L}(f) \frac{d t}{t^{-\alpha / 2+1}}(x) .
$$

Note that if $L=-\Delta$ is the Laplacian on $\mathbb{R}^{n}$, then $L^{-\alpha / 2}$ is the classical fractional integral $I_{\alpha}$ which plays important roles in many fields. It is well known that $I_{\alpha}$ is bounded from $L^{p}\left(\mathbb{R}^{n}\right)$ to $L^{q}\left(\mathbb{R}^{n}\right)$ for all $p>1,1 / q=1 / p-\alpha / n>0$ and is also of weak type $(1, n /(n-\alpha))$. 
Let $1 \leq p<\infty$ and $\omega$ be a weight function. A locally integrable function $b$ is said to be in $\mathrm{BMO}_{p}(\omega)$ if

$$
\begin{aligned}
\|b\|_{\mathrm{BMO}_{p}(\omega)} & =\sup _{\mathrm{Q}}\left(\frac{1}{\omega(Q)} \int_{\mathrm{Q}}\left|b(x)-b_{\mathrm{Q}}\right|^{p} \omega(x)^{1-p} d x\right)^{1 / p} \\
& \leq C<\infty,
\end{aligned}
$$

where $b_{\mathrm{Q}}=(1 /|\mathrm{Q}|) \int_{\mathrm{Q}} b(y) d y$ and the supremum is taken over all balls $Q \in \mathbb{R}^{n}$.

Let $\omega \in A_{1}$; García-Cuerva [6] proved that the spaces $\mathrm{BMO}_{p}(\omega)$ coincide, and the norms of $\|\cdot\|_{\mathrm{BMO}_{p}(\omega)}$ are equivalent with respect to different values provided that $1 \leq p<\infty$.

Let $b$ be a locally integrable function on $\mathbb{R}^{n}$; we consider the commutator $\left[b, L^{-\alpha / 2}\right]$ defined by

$$
\left[b, L^{-\alpha / 2}\right](f)(x)=b(x) L^{-\alpha / 2}(f)(x)-L^{-\alpha / 2}(b f)(x) .
$$

Chanillo [7] proved that the commutator $\left[b, I_{\alpha}\right]$ of the multiplication operator by $b \in \mathrm{BMO}$ is bounded on $L^{p}$ for $1<p<\infty$.

Duong and Yan [8] proved that $\left[b, L^{-\alpha / 2}\right]$ is bounded from $L^{p}$ to $L^{q}$, where $b \in \mathrm{BMO}, 1<p<n / \alpha, 1 / q=1 / p-\alpha / n, 0<$ $\alpha<n$.

Mo and $\mathrm{Lu}$ [9] proved that the multilinear commutator generated by $\vec{b}$ and $L^{-\alpha / 2}$ is bounded from $L^{p}$ to $L^{q}$, where $1<$ $p<n / \alpha, 1 / q=1 / p-\alpha / n, 0<\alpha<1, \vec{b}=\left(b_{1}, \ldots, b_{m}\right), b_{i} \in$ BMO, for $i=1, \ldots, m$.

Lu et al. [10] proved that $\left[b, I_{\alpha}\right]$ is bounded from $L^{p}$ to $L^{q}$ if and only if $b \in \mathrm{BMO}$.

Wang [11] proved that $\left[b, I_{\alpha}\right]$ is bounded from $L^{p, k}(\omega)$ to $L^{q, k q / p}\left(\omega^{1-(1-\alpha / n) q}, \omega\right)$, where $b \in \operatorname{BMO}(\omega), 0<\alpha<n, 1<$ $p<n / \alpha, 1 / q=1 / p-\alpha / n, 0<k<p / q$, and $\omega^{q / p} \in A_{1}$.

Inspired by the above results, we study the boundedness properties of the commutator $\left[b, L^{-\alpha / 2}\right]$ on weighted Morrey spaces in this work. The main theorems are stated as follows.

Theorem 1. Let $0<\alpha<n, 1<p<n / \alpha, 1 / q=1 / p-$ $\alpha / n, 0 \leq k<p / q, \omega^{q / p} \in A_{1}$, and $r_{\omega}>((1-k) /(p / q-k))$, where $r_{\omega}$ denotes the critical index of $\omega$ for the reverse Hölder condition. Then the following conditions are equivalent.

(a) $b \in B M O(\omega)$.

(b) $\left[b, L^{-\alpha / 2}\right]$ is bounded from $L^{p, k}(\omega)$ to $L^{q, k q / p}\left(\omega^{1-(1-\alpha / n) q}, \omega\right)$.

In particular, when $k=0$ in Theorem 1, we get the following.

Corollary 2. Let $0<\alpha<n, 1<p<n / \alpha, 1 / q=1 / p-$ $\alpha / n, \omega^{q / p} \in A_{1}$, and $r_{\omega}>q / p$, where $r_{\omega}$ denotes the critical index of $\omega$ for the reverse Hölder condition. Then the following conditions are equivalent.

(a) $b \in B M O(\omega)$.

(b) $\left[b, L^{-\alpha / 2}\right]$ is bounded from $L^{p}(\omega)$ to $L^{q}\left(\omega^{1-(1-\alpha / n) q}\right)$.
Furthermore, if $L=-\Delta$ is the Laplacian, then the following conditions are equivalent.

$\left(\mathrm{a}^{\prime}\right) b \in B M O(\omega)$.

$\left(\mathrm{b}^{\prime}\right)\left[b, I_{\alpha}\right]$ is bounded from $L^{p}(\omega)$ to $L^{q}\left(\omega^{1-(1-\alpha / n) q}\right)$.

Theorem 3. Let $0<\alpha<n, 0 \leq k<p / q, 1 / q=1 / p-\alpha / n$, and $1<r, s<\infty$ such that $1<r s<p<n / \alpha, \omega^{r s} \in A_{p / r s, q / r s}$. Then the following conditions are equivalent.

(a) $b \in B M O$.

(b) $\left[b, L^{-\alpha / 2}\right]$ is bounded from $L^{p, k}\left(\omega^{p}, \omega^{q}\right)$ to $L^{q, k q / p}\left(\omega^{q}\right)$.

In particular, when $k=0$ in Theorem 3, we obtain the following.

Corollary 4. Let $0<\alpha<n, 1 / q=1 / p-\alpha / n$, and $1<r, s<$ $\infty$ such that $1<r s<p<n / \alpha, \omega^{r s} \in A_{p / r s, q / r s}$. Then the following conditions are equivalent.

(a) $b \in B M O$.

(b) $\left[b, L^{-\alpha / 2}\right]$ is bounded from $L^{p}\left(\omega^{p}\right)$ to $L^{q}\left(\omega^{q}\right)$.

Furthermore, if $L=-\Delta$ is the Laplacian, then the following conditions are equivalent.

$\left(\mathrm{a}^{\prime}\right) b \in B M O$.

$\left(b^{\prime}\right)\left[b, I_{\alpha}\right]$ is bounded from $L^{p}\left(\omega^{p}\right)$ to $L^{q}\left(\omega^{q}\right)$.

Remark 5. It is easy to see that our results extend the results in $[7,8,10,11]$ significantly.

\section{Prerequisite Material}

Let us first recall some definitions.

Definition 6. The Hardy-Littlewood maximal operator $M$ is defined by

$$
M(f)(x)=\sup _{x \in Q} \frac{1}{|Q|} \int_{Q}|f(y)| d y .
$$

Let $\omega$ be a weight. The weighted maximal operator $M_{\omega}$ is defined by

$$
M_{\omega}(f)(x)=\sup _{x \in Q} \frac{1}{\omega(Q)} \int_{Q}|f(y)| \omega(y) d y .
$$

A variant of this maximal operator will become the main tool in our scheme; for $1<r<\infty$,

$$
M_{r, \omega}(f)(x)=M_{\omega}\left(|f|^{r}\right)^{1 / r}(x) .
$$

For $0<\alpha<n, r \geq 1$, the fractional maximal operator $M_{\alpha, r}$ is defined by

$$
M_{\alpha, r}(f)(x)=\sup _{x \in Q}\left(\frac{1}{|Q|^{1-\alpha r / n}} \int_{Q}|f(y)|^{r} d y\right)^{1 / r},
$$


and the fractional weighted maximal operator $M_{\alpha, r, \omega}$ is defined by

$$
M_{\alpha, r, \omega}(f)(x)=\sup _{x \in Q}\left(\frac{1}{\omega(Q)^{1-\alpha r / n}} \int_{Q}|f(y)|^{r} \omega(y) d y\right)^{1 / r} .
$$

For any $f \in L^{p}\left(\mathbb{R}^{n}\right), p \geq 1$, the sharp maximal function $M_{L}^{\sharp} f$ associated with the generalized approximations to the identity $\left\{e^{-t L}\right\}_{t>0}$ is given by

$$
M_{L}^{\sharp} f(x)=\sup _{x \in Q} \frac{1}{|Q|} \int_{Q}\left|f(y)-e^{-t_{\mathrm{Q}} L} f(y)\right| d y,
$$

where $t_{Q}=r_{Q}^{2}$ and $r_{Q}$ is the radius of the ball $Q$.

In the above definitions, the supremum is taken over all balls $Q$ containing $x$.

Definition 7. A weight function $\omega$ is said to be in the Muckenhoupt class $A_{p}$ with $1<p<\infty$ if, for every ball $Q$ in $\mathbb{R}^{n}$, there exists a positive constant $C$ which is independent of $Q$ such that

$$
\left(\frac{1}{|Q|} \int_{Q} \omega(x) d x\right)\left(\frac{1}{|Q|} \int_{Q} \omega(x)^{-1 /(p-1)} d x\right)^{p-1} \leq C .
$$

When $p=1, \omega \in A_{1}$, if

$$
\left(\frac{1}{|Q|} \int_{Q} \omega(x) d x\right) \leq C \text { ess } \inf _{x \in Q} \omega(x) .
$$

When $p=\infty, \omega \in A_{\infty}$, if there exist positive constants $\delta$ and $C$ such that, given a ball $Q$ and a measurable subset $E$ of $Q$,

$$
\frac{\omega(E)}{\omega(Q)} \leq C\left(\frac{|E|}{|Q|}\right)^{\delta}
$$

Definition 8. A weight function $\omega$ belongs to $A_{p, q}$ for $1<$ $p<q<\infty$ if, for every ball $Q$ in $\mathbb{R}^{n}$, there exists a positive constant $C$ which is independent of $Q$ such that

$$
\left(\frac{1}{|Q|} \int_{Q} \omega(x)^{q} d x\right)^{1 / q}\left(\frac{1}{|Q|} \int_{Q} \omega(x)^{-p^{\prime}} d x\right)^{1 / p^{\prime}} \leq C,
$$

where $p^{\prime}$ denotes the conjugate exponent of $p>1$, that is, $1 / p+1 / p^{\prime}=1$.

Definition 9. A weight function $\omega$ belongs to the reverse Hölder class $\mathrm{RH}_{r}$ if there exist two constants $r>1$ and $C>0$ such that the reverse Hölder inequality

$$
\left(\frac{1}{|Q|} \int_{Q} \omega(x)^{r} d x\right)^{1 / r} \leq C\left(\frac{1}{|Q|} \int_{Q} \omega(x) d x\right)
$$

holds for every ball $Q$ in $\mathbb{R}^{n}$.

It is well known that if $\omega \in A_{p}$ with $1 \leq p<\infty$, then there exists $r>1$ such that $\omega \in \mathrm{RH}_{r}$. It follows from Hölder's inequality that $\omega \in \mathrm{RH}_{r}$ implies $\omega \in \mathrm{RH}_{s}$ for all $1<s<r$. Moreover, if $\omega \in \mathrm{RH}_{r}, r>1$, then we have $\omega \in \mathrm{RH}_{r+\epsilon}$ for some $\epsilon>0$. We thus write $r_{w}=\sup \{r>1: \omega \epsilon$ $\left.\mathrm{RH}_{r}\right\}$ to denote the critical index of $\omega$ for the reverse Hölder condition.

We will make use of the following lemmas. We first provide a weighted version of the local good $\lambda$ inequality for $M_{L}^{\sharp}$ which allows us to obtain an analog of the classical Fefferman-Stein (see $[3,12]$ ) estimate on weighted Morrey spaces.

Lemma 10 (see [13]). Assume that the semigroup $e^{-t L}$ has a kernel $p_{t}(x, y)$ which satisfies the upper bound (3). Take $\lambda>$ $0, f \in L_{0}^{1}\left(\mathbb{R}^{n}\right)$, and a ball $Q_{0}$ such that there exists $x_{0} \in Q_{0}$ with $M f\left(x_{0}\right) \leq \lambda$. Then, for every $\omega \in A_{\infty}, 0<\eta<1$, one can find $\gamma>0$ (independent of $\lambda, Q_{0}, f, x_{0}$ ) and constant $C_{\omega}, r>$ 0 (which only depend on $\omega$ ), such that

$$
\omega\left\{x \in Q_{0}: M f(x)>A \lambda, M_{L}^{\sharp} f(x) \leq \gamma \lambda\right\} \leq C_{\omega} \eta^{r} \omega\left(Q_{0}\right),
$$

where $A>1$ is a fixed constant which depends only on $n$.

As a consequence, by using the standard arguments, we have the following estimates.

For every $f \in L^{p, k}(\mu, v)$, with $1<p<\infty$. if $\mu, v \in$ $A_{\infty}, 1<p<\infty, 0 \leq k<1$, then

$$
\|f\|_{L^{p, k}(\mu, \nu)} \leq\|M f\|_{L^{p, k}(\mu, \nu)} \leq C\left\|M_{L}^{\sharp} f\right\|_{L^{p, k}(\mu, \nu)} .
$$

In particular, when $\mu=\nu=\omega$ and $\omega \in A_{\infty}$, we have

$$
\|f\|_{L^{p, k}(\omega)} \leq\|M f\|_{L^{p, k}(\omega)} \leq C\left\|M_{L}^{\sharp} f\right\|_{L^{p, k}(\omega)} .
$$

Lemma 11 (see [11]). Let $0<\alpha<n, 1<p<n / \alpha, 1 / q=$ $1 / p-\alpha / n$, and $\omega^{q / p} \in A_{1}$. Then if $0<k<p / q$ and $r_{\omega}>$ $(1-k) /(p / q-k)$, one has

$$
\left\|M_{\alpha, 1} f\right\|_{L^{q, k q / p}\left(\omega^{q / p, \omega)}\right.} \leq C\|f\|_{L^{p, k}(\omega)} .
$$

The same conclusion still holds for $I_{\alpha}$.

Lemma 12 (see [11]). Let $0<\alpha<n, 1<p<n / \alpha, 1 / q=$ $1 / p-\alpha / n$, and $\omega^{q / p} \in A_{1}$. Then if $0<k<p / q, 1<r<p$, and $r_{\omega}>(1-k) /(p / q-k)$, one has

$$
\left\|M_{r, \omega} f\right\|_{L^{q, k q / p}\left(\omega^{q / p}, \omega\right)} \leq C\|f\|_{L^{q, k q / p}\left(\omega^{q / p}, \omega\right)} .
$$

Lemma 13 (see [11]). Consider $0<\alpha<n, 1<p<$ $n / \alpha, 1 / q=1 / p-\alpha / n, 0<k<p / q$, and $\omega \in A_{\infty}$. For any $1<r<p$, one has

$$
\left\|M_{\alpha, r, \omega} f\right\|_{L^{q, k q / p}(\omega)} \leq C\|f\|_{L^{p, k}(\omega)} .
$$

Remark 14. By checking the proof of Lemmas 11-13, we know that the three lemmas above still hold when $k=0$.

Lemma 15. Let $0<\alpha<n, 1<p<n / \alpha, 1 / q=1 / p-\alpha / n$, and $\omega^{q / p} \in A_{1}$. Then if $0 \leq k<p / q$ and $r_{\omega}>(1-k) /(p / q-k)$, one has

$$
\left\|L^{-\alpha / 2} f\right\|_{L^{q, k q / p}\left(\omega^{q / p}, \omega\right)} \leq C\|f\|_{L^{p, k}(\omega)}
$$


Proof. Since the semigroup $e^{-t L}$ has a kernel $p_{t}(x, y)$ which satisfies the upper bound (3), it is easy to check that $L^{-\alpha / 2}(f)(x) \leq C I_{\alpha}(|f|)(x)$ for all $x \in \mathbb{R}^{n}$. Using the boundedness property of $I_{\alpha}$ on weighted Morrey space (see Lemma 11), we have

$$
\left\|L^{-\alpha / 2} f\right\|_{L^{q, k q / p}\left(\omega^{q / p}, \omega\right)} \leq\left\|I_{\alpha} f\right\|_{L^{q, k q / p}\left(\omega^{q / p}, \omega\right)} \leq C\|f\|_{L^{p, k}(\omega)},
$$

where $1<p<n / \alpha$ and $1 / q=1 / p-\alpha / n$.

Remark 16. Since $I_{\alpha}$ is of weak type $(1, n /(n-\alpha))$. from the proof of Lemma 15, we can get that $L^{-\alpha / 2}$ is also of weak type $(1, n /(n-\alpha))$.

Lemma 17 (see $[8,14])$. Assume that the semigroup $e^{-t L}$ has a kernel $p_{t}(x, y)$ which satisfies the upper bound (3). Then for $0<\alpha<n$, the difference operator $L^{-\alpha / 2}-e^{-t L} L^{-\alpha / 2}$ has an associated kernel $K_{\alpha, t}(x, y)$ which satisfies

$$
\left|K_{\alpha, t}(x, y)\right| \leq \frac{C}{|x-y|^{n-\alpha}} \frac{t}{|x-y|^{2}},
$$

for some positive constant $C$.

Lemma 18. Assume that the semigroup $e^{-t L}$ has a kernel $p_{t}(x, y)$ which satisfies the upper bound (3), and let $b \in$ $B M O(\omega), \omega \in A_{1}$. Then, for every function $f \in L^{p}\left(\mathbb{R}^{n}\right), p>$ 1 , and for all $x \in \mathbb{R}^{n}$, one has

$$
\begin{array}{r}
\sup _{x \in Q} \frac{1}{|Q|} \int_{Q}\left|e^{-t_{Q} L}\left(b(y)-b_{Q}\right) f(y)\right| d y \\
\leq C\|b\|_{B M O(\omega)} \omega(x) M_{r, \omega}(f)(x),
\end{array}
$$

where $t_{\mathrm{Q}}=r_{\mathrm{Q}}^{2}, r_{\mathrm{Q}}$ being the radius of $\mathrm{Q}$.

Proof. For any $f \in L^{p}\left(\mathbb{R}^{n}\right), 1<p<\infty$ and $x \in Q$. We have

$$
\begin{aligned}
& \frac{1}{|Q|} \int_{Q}\left|e^{-t_{Q} L}\left(\left(b(\cdot)-b_{\mathrm{Q}}\right) f\right)(y)\right| d y \\
& \leq \frac{1}{|Q|} \int_{\mathrm{Q}} \int_{\mathbb{R}^{n}}\left|p_{t_{\mathrm{Q}}}(y, z)\right|\left|\left(b(z)-b_{\mathrm{Q}}\right) f(z)\right| d z d y \\
& \leq \frac{1}{|Q|} \int_{\mathrm{Q}} \int_{2 \mathrm{Q}}\left|p_{t_{\mathrm{Q}}}(y, z)\right|\left|\left(b(z)-b_{\mathrm{Q}}\right) f(z)\right| d z d y \\
& \quad+\frac{1}{|Q|} \int_{\mathrm{Q}^{2}} \sum_{k=1}^{\infty} \int_{2^{k+1} \mathrm{Q} \mid 2^{k} \mathrm{Q}}\left|p_{t_{\mathrm{Q}}}(y, z)\right| \\
& \times\left|\left(b(z)-b_{\mathrm{Q}}\right) f(z)\right| d z d y
\end{aligned}
$$$$
\doteq \mathbf{M}+\mathbf{N} \text {. }
$$

For any $y \in Q$ and $z \in 2 Q$. We have

$$
\left|p_{t_{\mathrm{Q}}}(y, z)\right| \leq C t_{\mathrm{Q}}^{-n / 2} \leq C \frac{1}{|2 Q|} .
$$

Thus,

$$
\begin{aligned}
\mathbf{M} \leq & C \frac{1}{|2 Q|} \int_{2 Q}\left|\left(b(z)-b_{\mathrm{Q}}\right) f(z)\right| d z \\
\leq & C \frac{1}{|2 Q|}\left(\int_{2 Q}\left|b(z)-b_{\mathrm{Q}}\right|^{r^{\prime}} \omega(z)^{1-r^{\prime}} d z\right)^{1 / r^{\prime}} \\
& \times\left(\int_{2 \mathrm{Q}}|f(z)|^{r} \omega(z) d z\right)^{1 / r} \\
\leq & C\|b\|_{\mathrm{BMO}(\omega)} \frac{\omega(2 Q)}{|2 Q|}\left(\frac{1}{\omega(2 Q)} \int_{2 \mathrm{Q}}|f(z)|^{r} \omega(z) d z\right)^{1 / r} \\
\leq & C\|b\|_{\mathrm{BMO}(\omega)} \omega(x) M_{r, \omega} f(x) .
\end{aligned}
$$

Moreover, for any $y \in Q$ and $z \in 2^{k+1} Q \backslash 2^{k} Q$, we have $|y-z| \geq$ $2^{k-1} r_{\mathrm{Q}}$ and $\left|p_{t_{\mathrm{Q}}}\right| \leq C\left(e^{-c 2^{2(k-1)}} 2^{(k+1) n} /\left|2^{k+1} \mathrm{Q}\right|\right)$ :

$$
\begin{aligned}
& \mathbf{N} \\
& =\frac{1}{|Q|} \int_{Q} \sum_{k=1}^{\infty} \int_{2^{k+1} Q 2^{k} Q}\left|p_{t_{Q}}(y, z)\right|\left|\left(b(z)-b_{Q}\right) f(z)\right| d z d y \\
& \leq C \sum_{k=1}^{\infty} \frac{e^{-c 2^{2(k-1)}} 2^{(k+1) n}}{\left|2^{k+1} Q\right|} \int_{2^{k+1} Q}\left|\left(b(z)-b_{Q}\right) f(z)\right| d z \\
& \leq C \sum_{k=1}^{\infty} \frac{e^{-c 2^{2(k-1)}} 2^{(k+1) n}}{\left|2^{k+1} Q\right|} \int_{2^{k+1} Q}\left|\left(b(z)-b_{2^{k+1} Q}\right) f(z)\right| d z \\
& \quad+C \sum_{k=1}^{\infty} \frac{e^{-c 2^{2(k-1)}} 2^{(k+1) n}}{\left|2^{k+1} Q\right|} \int_{2^{k+1} Q}\left|\left(b_{2^{k+1} Q}-b_{Q}\right) f(z)\right| d z \\
& \doteq \mathbf{N}_{1}+\mathbf{N}_{2} .
\end{aligned}
$$

We estimate each term in turn. For $\mathbf{N}_{1}$, we apply Hölder's inequalities with exponent $r$. Then we have

$$
\begin{aligned}
\mathbf{N}_{1} \leq & C \sum_{k=1}^{\infty} \frac{e^{-c 2^{2(k-1)}} 2^{(k+1) n}}{\left|2^{k+1} Q\right|} \\
& \times\left(\int_{2^{k+1} Q}\left|b(z)-b_{2^{k+1}}\right|^{r^{\prime}} \omega(z)^{1-r^{\prime}} d z\right)^{1 / r^{\prime}} \\
& \times\left(\int_{2^{k+1} Q}|f(z)|^{r} \omega(z) d z\right)^{1 / r} \\
\leq & C \sum_{k=1}^{\infty} 2^{(k+1) n} e^{-c 2^{2(k-1)}}\|b\|_{\mathrm{BMO}(\omega)} \frac{\omega\left(2^{k+1} Q\right)}{\left|2^{k+1} Q\right|} \\
& \times\left(\frac{1}{\omega\left(2^{k+1} Q\right)} \int_{2^{k+1} Q}|f(z)|^{r} \omega(z) d z\right)^{1 / r} \\
\leq & C\|b\|_{\mathrm{BMO}(\omega)} \omega(x) M_{r, \omega} f(x) .
\end{aligned}
$$


Since $\omega \in A_{1}$, then $\left|b_{2^{k+1 Q}}-b_{\mathrm{Q}}\right| \leq C k \omega(x)\|b\|_{\mathrm{BMO}(\omega)}$. This fact together with Hölder's inequality implies

$$
\begin{aligned}
\mathbf{N}_{2} \leq & C \sum_{k=1}^{\infty} 2^{(k+1) n} e^{-c 2^{2(k-1)}} \frac{k}{\left|2^{k+1} Q\right|} \omega(x) \\
& \times\|b\|_{\mathrm{BMO}(\omega)} \int_{Q}|f(z)| d z \\
\leq & C \sum_{k=1}^{\infty} k 2^{(k+1) n} e^{-c 2^{2(k-1)}} \omega(x) \\
& \times\|b\|_{\mathrm{BMO}(\omega)}\left(\frac{1}{\left|2^{k+1} Q\right|} \int_{2^{k+1} Q}|f(z)|^{r} d z\right)^{1 / r} \\
= & C \sum_{k=1}^{\infty} k 2^{(k+1) n} e^{-c 2^{2(k-1)}} \omega(x) \\
& \times\|b\|_{\mathrm{BMO}(\omega)}\left(\frac{\omega\left(2^{k+1} Q\right)}{\left|2^{k+1} Q\right|} \frac{1}{\omega\left(2^{k+1} Q\right)} \int_{2^{k+1} Q}|f(z)|^{r} d z\right)^{1 / r} \\
\leq & C \sum_{k=1}^{\infty} k 2^{(k+1) n} e^{-c 2^{2(k-1)}} \omega(x) \\
& \times\|b\|_{\mathrm{BMO}(\omega)}\left(\frac{1}{\omega\left(2^{k+1} Q\right)} \int_{2^{k+1} Q}|f(z)|^{r} \omega(x) d z\right)^{1 / r} \\
\leq & C\|b\|_{\mathrm{BMO}(\omega)} \omega(x) M_{r, \omega} f(x) .
\end{aligned}
$$

Then Lemma 18 is proved.

Lemma 19. Let $0<\alpha<n, \omega \in A_{1}$, and $b \in B M O(\omega)$. Then for all $r>1$ and for all $x \in \mathbb{R}^{n}$, one has

$$
\begin{aligned}
M_{L}^{\sharp}\left(\left[b, L^{-\alpha / 2}\right] f\right)(x) & \\
\leq C\|b\|_{B M O(\omega)}( & \left(\omega(x) M_{r, \omega}\left(L^{-\alpha / 2} f\right)(x)+\omega(x)^{1-\alpha / n}\right. \\
& \left.\times M_{\alpha, r, \omega}(f)(x)+\omega(x) M_{\alpha, 1}(f)(x)\right) .
\end{aligned}
$$

Proof. For any given $x \in \mathbb{R}^{n}$, fix a ball $Q=Q\left(x_{0}, r_{Q}\right)$ which contains $x$. We decompose $f=f_{1}+f_{2}$, where $f_{1}=f \chi_{2 Q}$. Observe that

$$
\begin{aligned}
{\left[b, L^{-\alpha / 2}\right] f(x)=} & \left(b-b_{\mathrm{Q}}\right) L^{-\alpha / 2} f \\
& -L^{-\alpha / 2}\left(b-b_{\mathrm{Q}}\right) f_{1} \\
& -L^{-\alpha / 2}\left(b-b_{\mathrm{Q}}\right) f_{2}, \\
e^{-t_{\mathrm{Q}} L}\left(\left[b, L^{-\alpha / 2}\right] f\right)= & e^{-t_{\mathrm{Q}} L}\left[\left(b-b_{\mathrm{Q}}\right) L^{-\alpha / 2} f\right. \\
& -L^{-\alpha / 2}\left(b-b_{\mathrm{Q}}\right) f_{1} \\
& \left.-L^{-\alpha / 2}\left(b-b_{\mathrm{Q}}\right) f_{2}\right] .
\end{aligned}
$$

Then

$$
\begin{aligned}
& \frac{1}{|Q|} \int_{Q}\left|\left[b, L^{-\alpha / 2}\right] f(y)-e^{-t_{Q} L}\left[b, L^{-\alpha / 2}\right] f(y)\right| d y \\
& \leq \frac{1}{|Q|} \int_{Q}\left|\left(b(y)-b_{\mathrm{Q}}\right) L^{-\alpha / 2} f(y)\right| d y \\
& \quad+\frac{1}{|Q|} \int_{Q}\left|L^{-\alpha / 2}\left(b-b_{\mathrm{Q}}\right) f_{1}(y)\right| d y \\
& \quad+\frac{1}{|\mathrm{Q}|} \int_{\mathrm{Q}}\left|e^{-t_{\mathrm{Q}} L}\left(\left(b-b_{\mathrm{Q}}\right) L^{-\alpha / 2} f\right)(y)\right| d y \\
& \quad+\frac{1}{|\mathrm{Q}|} \int_{\mathrm{Q}}\left|e^{-t_{\mathrm{Q}} L} L^{-\alpha / 2}\left(\left(b-b_{\mathrm{Q}}\right) f_{1}(y)\right)\right| d y \\
& \quad+\frac{1}{|\mathrm{Q}|} \int_{\mathrm{Q}}\left|\left(L^{-\alpha / 2}-e^{-t_{\mathrm{Q}} L} L^{-\alpha / 2}\right)\left(\left(b-b_{\mathrm{Q}}\right) f_{2}\right)(y)\right| d y \\
& \doteq \mathbf{I}+\mathbf{I I}+\mathbf{I I I}+\mathbf{I V}+\mathbf{V} .
\end{aligned}
$$

We estimate each term separately.

Since $\omega \in A_{1}$, then it follows from Hölder's inequality that

$$
\begin{aligned}
\mathbf{I} \leq & \frac{1}{|Q|} \int_{\mathrm{Q}}\left|\left(b(y)-b_{\mathrm{Q}}\right) L^{-\alpha / 2} f(y)\right| d y \\
\leq & \frac{1}{|Q|}\left(\int_{\mathrm{Q}}\left|b(y)-b_{\mathrm{Q}}\right|^{r^{\prime}} \omega(y)^{1-r^{\prime}} d y\right)^{1 / r^{\prime}} \\
& \times\left(\int_{\mathrm{Q}}\left|L^{-\alpha / 2} f(y)\right|^{r} \omega(y) d y\right)^{1 / r} \\
\leq & C\|b\|_{\mathrm{BMO}(\omega)} \frac{\omega(Q)}{|Q|}\left(\frac{1}{\omega(Q)} \int_{\mathrm{Q}}\left|L^{-\alpha / 2} f(y)\right|^{r} \omega(y) d y\right)^{1 / r} \\
\leq & C\|b\|_{\mathrm{BMO}(\omega)} \omega(x) M_{r, \omega}\left(L^{-\alpha / 2} f\right)(x) .
\end{aligned}
$$

Applying Kolmogorov's inequality (see [15, page 485]), Hölder's inequality, and the continuity of $L^{-\alpha / 2}$, we thus have

$$
\begin{aligned}
\mathbf{I I} & =\frac{1}{|Q|} \int_{\mathrm{Q}}\left|L^{-\alpha / 2}\left(b-b_{\mathrm{Q}}\right) f_{1}(y)\right| d y \\
& \leq C \frac{1}{|\mathrm{Q}|^{1-\alpha / n}}\left\|L^{-\alpha / 2}\left(b-b_{\mathrm{Q}}\right) f_{1}\right\|_{L^{n /(n-\alpha), \infty}} \\
& \leq C \frac{1}{|\mathrm{Q}|^{1-\alpha / n}} \int_{\mathrm{Q}}\left(b-b_{\mathrm{Q}}\right) f_{1}(y) d y \\
& \leq C \frac{1}{|\mathrm{Q}|^{1-\alpha / n}}\left(\int_{\mathrm{Q}}\left|b(y)-b_{\mathrm{Q}}\right|^{r^{\prime}} \omega(y)^{1-r^{\prime}} d y\right)^{1 / r^{\prime}}
\end{aligned}
$$




$$
\begin{aligned}
& \times\left(\int_{Q}|f(y)|^{r} \omega(y) d y\right)^{1 / r} \\
\leq & C\|b\|_{\mathrm{BMO}(\omega)} \frac{w(Q)^{1-\alpha / n}}{|Q|^{1-\alpha / n}} \\
& \times\left(\frac{1}{w(Q)^{1-r \alpha / n}} \int_{\mathrm{Q}}|f(y)|^{r} \omega(y) d y\right)^{1 / r} \\
\leq & C\|b\|_{\mathrm{BMO}(\omega)} \omega(x)^{1-\alpha / n} M_{\alpha, r, \omega}(f)(x) .
\end{aligned}
$$

By Lemma 18, we have

$$
\mathrm{III} \leq C\|b\|_{\mathrm{BMO}(\omega)} \omega(x) M_{r, \omega}\left(L^{-\alpha / 2} f\right)(x) .
$$

For IV, using the estimate obtained in II, we get

$$
\begin{aligned}
\mathbf{I V} & \leq \frac{1}{|Q|} \int_{\mathrm{Q}} \int_{2 \mathrm{Q}}\left|p_{t_{\mathrm{Q}}}(y, z)\right|\left|b(z)-b_{\mathrm{Q}}\right||f(z)| d z d y \\
& \leq \frac{1}{|2 Q|} \int_{2 \mathrm{Q}}\left|L^{-\alpha / 2}\left(\left(b(z)-b_{\mathrm{Q}}\right)\right) f(z)\right| d z \\
& \leq C\|b\|_{\mathrm{BMO}(\omega)} \omega(x)^{1-\alpha / n} M_{\alpha, r, \omega}(f)(x) .
\end{aligned}
$$

By virtue of Lemma 17, we have

$$
\begin{aligned}
\mathbf{V} \leq & \frac{1}{|Q|} \int_{\mathrm{Q}} \int_{(2 \mathrm{Q})^{c}}\left|K_{\alpha, t_{\mathrm{Q}}}(y, z)\right|\left|\left(b(z)-b_{\mathrm{Q}}\right) f(z)\right| d z d y \\
\leq & C \sum_{k=1}^{\infty} \int_{2^{k} r_{\mathrm{Q}} \leq\left|x_{0}-z\right|<2^{k+1} r_{\mathrm{Q}}} \frac{1}{\left|x_{0}-z\right|^{n-\alpha}} \frac{t_{\mathrm{Q}}}{\left|x_{0}-z\right|^{2}} \\
& \times\left|\left(b(z)-b_{\mathrm{Q}}\right) f(z)\right| d z \\
\leq & C \sum_{k=1}^{\infty} 2^{-2 k} \frac{1}{\left|2^{k+1} \mathrm{Q}\right|^{1-\alpha / n}} \int_{2^{k+1} \mathrm{Q}}\left|\left(b(z)-b_{\mathrm{Q}}\right) f(z)\right| d z \\
\leq & C \sum_{k=1}^{\infty} 2^{-2 k} \frac{1}{\left|2^{k+1} \mathrm{Q}\right|^{1-\alpha / n}} \int_{2^{k+1} \mathrm{Q}}\left|\left(b(z)-b_{2^{k+1} \mathrm{Q}}\right) f(z)\right| d z \\
& +C \sum_{k=1}^{\infty} 2^{-2 k}\left(b_{2^{k+1} \mathrm{Q}}-b_{\mathrm{Q}}\right) \frac{1}{\left|2^{k+1} \mathrm{Q}\right|^{1-\alpha / n}} \int_{2^{k+1} \mathrm{Q}}|f(z)| d z \\
\doteq & \mathrm{VI}+\mathbf{V I I} .
\end{aligned}
$$

For VI, applying the same arguments as in II, we get

$$
\begin{aligned}
\mathbf{V I} & \leq C\|b\|_{\mathrm{BMO}(\omega)} \sum_{k=1}^{\infty} 2^{-2 k} \omega(x)^{1-\alpha / n} M_{\alpha, r, \omega}(f)(x) \\
& \leq C\|b\|_{\mathrm{BMO}(\omega)} \omega(x)^{1-\alpha / n} M_{\alpha, r, \omega}(f)(x) .
\end{aligned}
$$

Since $\omega \in A_{1}$, then $\left|b_{2^{k+1 Q}}-b_{\mathrm{Q}}\right| \leq C k \omega(x)\|b\|_{\mathrm{BMO}(\omega)}$. Thus,

$$
\begin{aligned}
\mathbf{V I I} & \leq C\|b\|_{\mathrm{BMO}(\omega)} \sum_{k=1}^{\infty} 2^{-2 k} k \omega(x) M_{\alpha, 1}(f)(x) \\
& \leq C\|b\|_{\mathrm{BMO}(\omega)} \omega(x) M_{\alpha, 1}(f)(x) .
\end{aligned}
$$

Then

$$
\begin{aligned}
\mathbf{V} \leq & C\|b\|_{\mathrm{BMO}(\omega)} \\
& \times\left(\omega(x)^{1-\alpha / n} M_{\alpha, r, \omega}(f)(x)+\omega(x) M_{\alpha, 1}(f)(x)\right) .
\end{aligned}
$$

Combining the above estimates I-V, we get (34). The proof of Lemma 19 is complete.

\section{Proofs of the Main Results}

In this section we prove our main results. We start with the proof of Theorem 1 .

Proof. (a) $\Rightarrow(\mathrm{b})$ : Applying Lemmas 10 and 19, we get

$$
\begin{aligned}
\left\|\left[b, L^{-\alpha / 2}\right] f\right\|_{L^{q, k q / p}\left(\omega^{1-(1-\alpha / n) q}, \omega\right)} & \\
\leq & M_{L}^{\sharp}\left(\left[b, L^{-\alpha / 2}\right] f\right) \|_{L^{q, k q / p}\left(\omega^{1-(1-\alpha / n) q}, \omega\right)} \\
\leq & C\|b\|_{\mathrm{BMO}(\omega)} \\
& \times\left(\left\|\omega M_{r, \omega}\left(L^{-\alpha / 2} f\right)\right\|_{L^{q, k q / p}\left(\omega^{1-(1-\alpha / n) q}, \omega\right)}\right. \\
& +\left\|\omega^{1-\alpha / n} M_{\alpha, r, \omega} f\right\|_{L^{q, k q / p}\left(\omega^{1-(1-\alpha / n) q}, \omega\right)} \\
& \left.+\left\|\omega M_{\alpha, 1} f\right\|_{L^{q, k q / p}\left(\omega^{1-(1-\alpha / n) q}, \omega\right)}\right) \\
\leq & C\|b\|_{\mathrm{BMO}(\omega)} \\
\times & \left(\left\|M_{r, \omega}\left(L^{-\alpha / 2} f\right)\right\|_{L^{q, k q / p}\left(\omega^{q / p}, \omega\right)}\right. \\
& +\left\|M_{\alpha, r, \omega} f\right\|_{L^{q, k q / p}(\omega)} \\
& \left.+\left\|M_{\alpha, 1} f\right\|_{L^{q, k q / p}\left(\omega^{q / p}, \omega\right)}\right) .
\end{aligned}
$$

Since $0 \leq k<p / q, \omega^{q / p} \in A_{1}$, and $r_{\omega}>(1-k) /(p / q-k)$, by making use of Lemmas 11-13, then we obtain

$$
\begin{aligned}
& \left\|\left[b, L^{-\alpha / 2}\right] f\right\|_{L^{q, k q / p}\left(\omega^{1-(1-\alpha / n) q}, \omega\right)} \\
& \quad \leq C\|b\|_{\mathrm{BMO}(\omega)}\left(\left\|L^{-\alpha / 2} f\right\|_{L^{q, k q / p}\left(\omega^{q / p}, \omega\right)}+\|f\|_{L^{p, k}(\omega)}\right) \\
& \quad \leq C\|b\|_{\mathrm{BMO}(\omega)}\|f\|_{L^{p, k}(\omega)} .
\end{aligned}
$$

The last inequality follows from Lemma 15. This completes the proof of $(a) \Rightarrow(b)$.

(b) $\Rightarrow$ (a): Let $L=-\Delta$ be the Laplacian on $\mathbb{R}^{n}$; then $L^{-\alpha / 2}$ is the classical fractional integral $I_{\alpha}$. Choose $Z_{0} \in \mathbb{R}^{n}$ so that $\left|Z_{0}\right|=3$. For $x \in Q\left(Z_{0}, 2\right),|x|^{-\alpha+n}$ can be written as the absolutely convergent Fourier series $|x|^{-\alpha+n}=\sum_{m \in Z_{n}} a_{m} e^{i\left\langle v_{m}, x\right\rangle}$ 
with $\sum_{m}\left|a_{m}\right|<\infty$ since $|x|^{-\alpha+n} \in C^{\infty}\left(Q\left(Z_{0}, 2\right)\right)$. For any $x_{0} \in \mathbb{R}^{n}$ and $\rho>0$, let $Q=Q\left(x_{0}, \rho\right)$ and $Q_{Z_{0}}=Q\left(x_{0}+Z_{0} \rho, \rho\right)$,

$$
\begin{aligned}
& \int_{Q}\left|b(x)-b_{\mathrm{Q}_{0}}\right| d x \\
& =\frac{1}{\left|Q_{Z_{0}}\right|} \int_{Q}\left|\int_{Q_{Z_{0}}}(b(x)-b(y)) d y\right| d x \\
& =\frac{1}{\rho^{n}} \int_{Q} s(x)\left(\int_{Q_{Z_{0}}}(b(x)-b(y))|x-y|^{\alpha-n}|x-y|^{n-\alpha} d y\right) d x,
\end{aligned}
$$

where $s(x)=\operatorname{sgn}\left(\int_{Q_{Z_{0}}}(b(x)-b(y)) d y\right)$. Fix $x \in Q$ and $y \in Q_{Z_{0}}$ and we have $(y-x) / \rho \in Q\left(Z_{0}, 2\right)$; hence, we have

$$
\begin{aligned}
& \frac{\rho^{-\alpha+n}}{\rho^{n}} \int_{Q} s(x)\left(\int_{Q_{Z_{0}}}(b(x)-b(y))|x-y|^{\alpha-n}\left(\frac{|x-y|}{\rho}\right)^{n-\alpha} d y\right) d x \\
& =\rho^{-\alpha} \sum_{m \in Z^{n}} a_{m} \\
& \times \int_{Q} s(x)\left(\int_{Q_{Z_{0}}}(b(x)-b(y))\right. \\
& \left.\times|x-y|^{\alpha-n} e^{i\left\langle v_{m}, y / \rho\right\rangle} d y\right) e^{-i\left\langle v_{m}, x / \rho\right\rangle} d x \\
& \leq \rho^{-\alpha}\left|\sum_{m \in Z^{n}}\right| a_{m} \mid \int_{Q} s(x)\left[b, L^{-\alpha / 2}\right] \\
& \times\left(\chi_{\mathrm{Q}_{0}} e^{i\left\langle v_{m}, / \rho\right\rangle}\right) \chi_{\mathrm{Q}}(x) e^{-i\left\langle v_{m}, x / \rho\right\rangle} d x \\
& \leq \rho^{-\alpha} \sum_{m \in Z^{n}}\left|a_{m}\right|\left\|\left[b, L^{-\alpha / 2}\right]\left(\chi_{\mathrm{Q}_{Z_{0}}} e^{i\left\langle v_{m}, / \rho\right\rangle}\right)\right\|_{L^{q, 0}\left(\omega^{1-(1-(\alpha / n)) q, \omega)}\right.} \\
& \times\left(\int_{Q} \omega(x)^{q^{\prime}[(1-(\alpha / n))-1 / q]} d x\right)^{1 / q^{\prime}} \\
& \leq C \rho^{-\alpha} \sum_{m \in Z^{n}}\left|a_{m}\right|\left\|\chi_{\mathrm{Q}_{0}}\right\|_{L^{p, 0}(\omega)}\left(\int_{Q} \omega(x)^{q^{\prime}\left(1 / q^{\prime}-\alpha / n\right)} d x\right)^{1 / q^{\prime}} \\
& \leq C \omega(Q)^{1 / p+1 / q^{\prime}-\alpha / n} \\
& =C \omega(Q) \text {. }
\end{aligned}
$$

This implies $b \in \mathrm{BMO}(\omega)$. Thus Theorem 1 is proved.

Similarly, to prove Theorem 3, we need the following lemmas.

Lemma 20. Let $0<\alpha<n, 1<r, s<\infty$ such that $r s<p<$ $n / \alpha$ and $b \in B M O$. Then for all $r>1$ and for all $x \in \mathbb{R}^{n}$, one has

$$
\begin{aligned}
& M_{L}^{\sharp}\left(\left[b, L^{-\alpha / 2}\right] f\right)(x) \\
& \quad \leq C\|b\|_{B M O}\left(M_{r}\left(L^{-\alpha / 2} f\right)(x)+M_{\alpha, r s}(f)(x)\right),
\end{aligned}
$$

where $M_{r}(f)(x)=M\left(|f|^{r}\right)^{1 / r}(x)$.
Proof. The case $0<\alpha<1$ was proved by Duong and Yan (see [8] for details). The general case $0<\alpha<n$ follows by repeating the same steps as in Lemma 19. Since the main steps and the ideas are almost the same, here we omit the proof.

Lemma 21 (see [5]). If $0<\alpha<n, 1<p<n / \alpha, 1 / q=1 / p-$ $\alpha / n, 0<k<p / q$, and $\omega \in A_{p, q}$, then the fractional maximal operator $M_{\alpha, 1}$ is bounded from $L^{p, k}\left(\omega^{p}, \omega^{q}\right)$ to $L^{q, k q / p}\left(\omega^{q}\right)$.

Lemma 22 (see [5]). If $0<\alpha<n, 1<p<n / \alpha, 1 / q=1 / p-$ $\alpha / n, 0<k<p / q$, and $\omega \in A_{p, q}$, then the fractional maximal operator $I_{\alpha}$ is bounded from $L^{p, k}\left(\omega^{p}, \omega^{q}\right)$ to $L^{q, k q / p}\left(\omega^{q}\right)$.

Lemma 23 (see [5]). If $1<p<\infty, 0<k<1$, and $\omega \in A_{p}$, then $M$ is bounded on $L^{p, k}(\omega)$.

Remark 24. By applying the same argument as in Lemma 15, we know that the conclusion in Lemma 22 still holds for $L^{-\alpha / 2}$. We omit the proof here.

Remark 25. By checking the proof of Lemmas 21-23, we know that the three lemmas above still hold when $k=0$.

Now we prove Theorem 3.

Proof. (a) $\Rightarrow(\mathrm{b})$ : Since $\omega^{r s} \in A_{p / r s, q / r s}$, then we get $\omega^{q} \in A_{q / r s}$ and $\omega^{p} \in A_{p / r s}$. Applying Lemmas 10 and 20-23, we get

$$
\begin{aligned}
& \left\|\left[b, L^{-\alpha / 2}\right] f\right\|_{L^{q, k q / p}\left(\omega^{q}\right)} \\
& \quad \leq\left\|M_{L}^{\sharp}\left(\left[b, L^{-\alpha / 2}\right] f\right)\right\|_{L^{q, k q / p}\left(\omega^{q}\right)} \\
& \quad \leq C\|b\|_{\mathrm{BMO}}\left(\left\|M_{r}\left(L^{-\alpha / 2} f\right)\right\|_{L^{q, k q / p}\left(\omega^{q)}\right)}+\left\|M_{\alpha, r s}(f)\right\|_{L^{q, k q / p}\left(\omega^{q)}\right)}\right) \\
& \quad \leq C\|b\|_{\mathrm{BMO}}\left(\left\|L^{-\alpha / 2} f\right\|_{L^{q, k q / p}\left(\omega^{q}\right)}+\|f\|_{L^{p, k}\left(\omega^{p}, \omega^{q}\right)}\right) \\
& \quad \leq C\|b\|_{\mathrm{BMO}}\|f\|_{L^{p, k}\left(\omega^{p}, \omega^{q}\right)} .
\end{aligned}
$$

In the last inequality, we used the fact that $L^{-\alpha / 2}$ is bounded from $L^{p, k}\left(\omega^{p}, \omega^{q}\right)$ to $L^{q, k q / p}\left(\omega^{q}\right)$ (see Remark 24).

(b) $\Rightarrow$ (a): Let $L=-\Delta$ be the Laplacian on $\mathbb{R}^{n}$; then $L^{-\alpha / 2}$ is the classical fractional integral $I_{\alpha}$. Let $k=0$ and weight $\omega \equiv 1$, and then $L^{p, k}\left(\omega^{p}, \omega^{q}\right)=L^{p}$ and $L^{q, k q / p}\left(\omega^{q}\right)=L^{q}$. From $[10]$ we deduce that the $\left(L^{p}, L^{q}\right)$ boundedness of $\left[b, I_{\alpha}\right]$ implies $b \in \mathrm{BMO}$. Thus Theorem 3 is proved.

\section{Conflict of Interests}

The authors declare that there is no conflict of interests regarding the publication of this paper.

\section{Acknowledgments}

This work was supported by the National Natural Science Foundation of China (no. 11226102) and the Doctoral Foundation of Henan Polytechnic University (no. B2012-055). 


\section{References}

[1] C. B. Morrey, Jr., "On the solutions of quasi-linear elliptic partial differential equations," Transactions of the American Mathematical Society, vol. 43, no. 1, pp. 126-166, 1938.

[2] F. Chiarenza and M. Frasca, "Morrey spaces and Hardy-Littlewood maximal function," Rendiconti di Matematica e delle sue Applicazioni, vol. 7, no. 3-4, pp. 273-279, 1987.

[3] R. R. Coifman and C. Fefferman, "Weighted norm inequalities for maximal functions and singular integrals," Studia Mathematica, vol. 51, pp. 241-250, 1974.

[4] B. Muckenhoupt, "Weighted norm inequalities for the Hardy maximal function," Transactions of the American Mathematical Society, vol. 165, pp. 207-226, 1972.

[5] Y. Komori and S. Shirai, "Weighted Morrey spaces and a singular integral operator," Mathematische Nachrichten, vol. 282, no. 2, pp. 219-231, 2009.

[6] J. García-Cuerva, "Weighted $H^{p}$ spaces," Dissertationes Mathematicae, vol. 162, pp. 1-63, 1979.

[7] S. Chanillo, "A note on commutators," Indiana University Mathematics Journal, vol. 31, no. 1, pp. 7-16, 1982.

[8] X. T. Duong and L. X. Yan, "On commutators of fractional integrals," Proceedings of the American Mathematical Society, vol. 132, no. 12, pp. 3549-3557, 2004.

[9] H. Mo and S. Lu, "Boundedness of multilinear commutators of generalized fractional integrals," Mathematische Nachrichten, vol. 281, no. 9, pp. 1328-1340, 2008.

[10] S. Lu, Y. Ding, and D. Yan, Singular Integrals and Related Topics, World Scientific Publishing, Hackensack, NJ, USA, 2007.

[11] H. Wang, "On some commutator theorems for fractional integ-ral operators on the weighted morreyspaces," http://arxiv-web3.library.cornell.edu/abs/1010.2638vl.

[12] C. Fefferman and E. M. Stein, " $H^{p}$ spaces of several variables," Acta Mathematica, vol. 129, no. 3-4, pp. 137-193, 1972.

[13] J. M. Martell, "Sharp maximal functions associated with approximations of the identity in spaces of homogeneous type and applications," Studia Mathematica, vol. 161, no. 2, pp. 113-145, 2004.

[14] D. Deng, X. T. Duong, A. Sikora, and L. Yan, "Comparison of the classical BMO with the BMO spaces associated with operators and applications," Revista Mathemática Iberoamericana, vol. 24, no. 1, pp. 267-296, 2008.

[15] J. García-Cuerva and J. L. Rubio de Francia, Weighted Norm Inequalities and Related Topics, vol. 116 of North-Holland Mathematics Studies, North-Holland, Amsterdam, The Netherlands, 1985. 


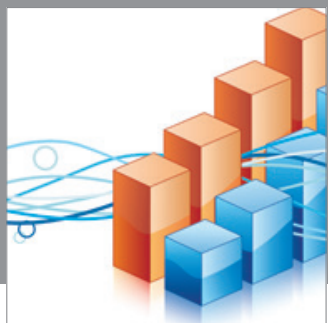

Advances in

Operations Research

mansans

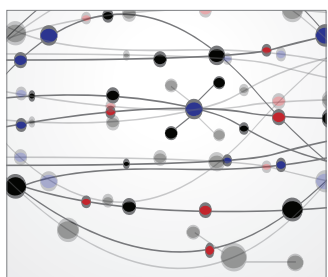

The Scientific World Journal
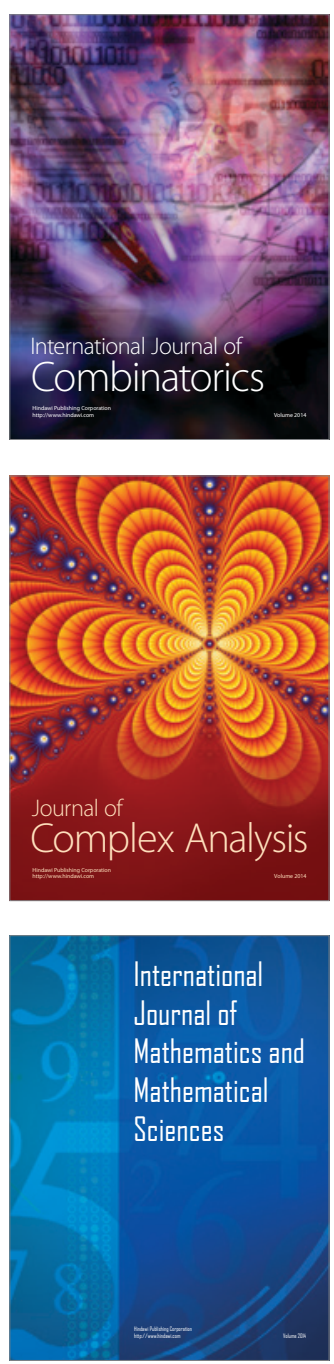
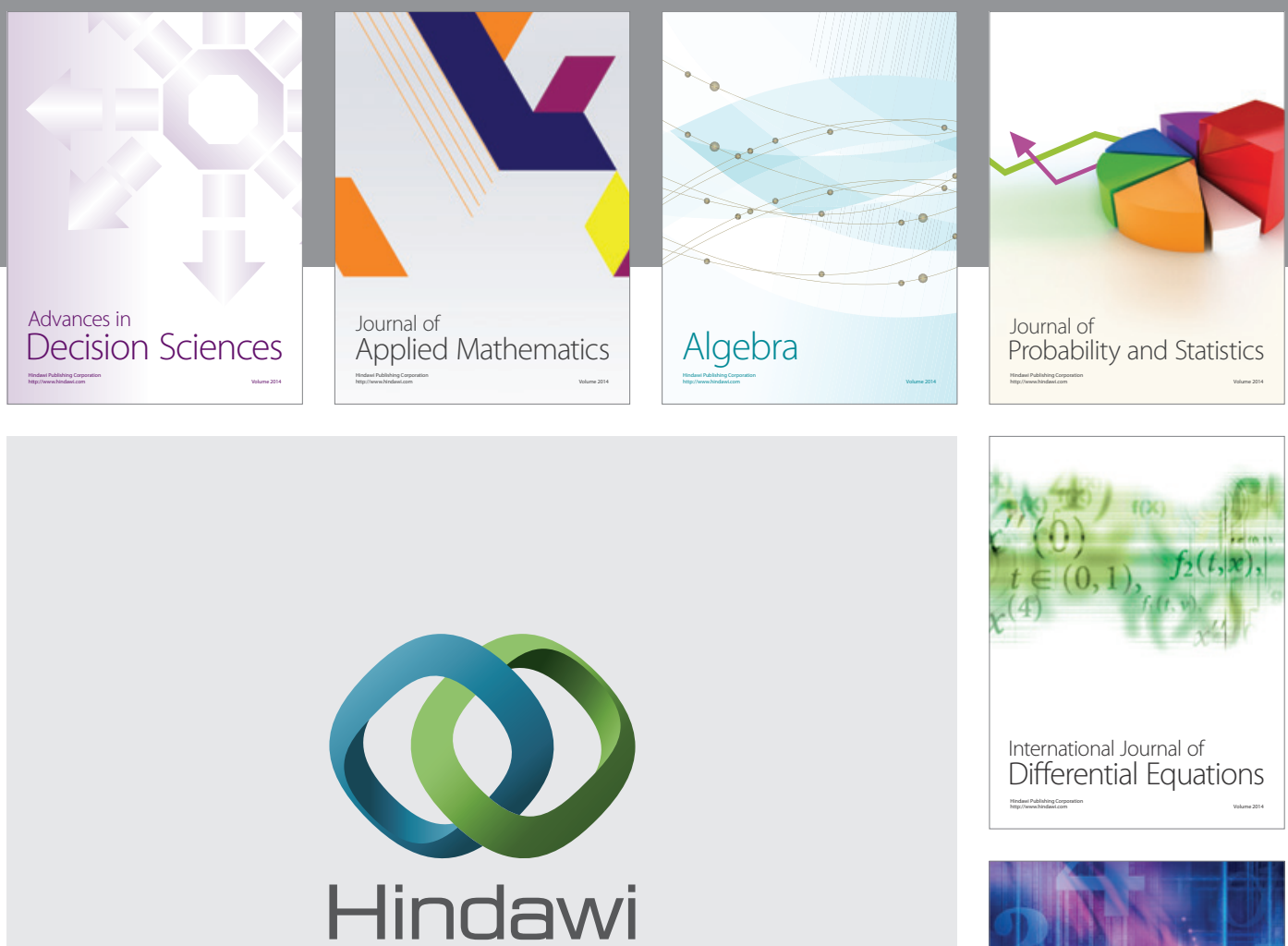

Submit your manuscripts at http://www.hindawi.com
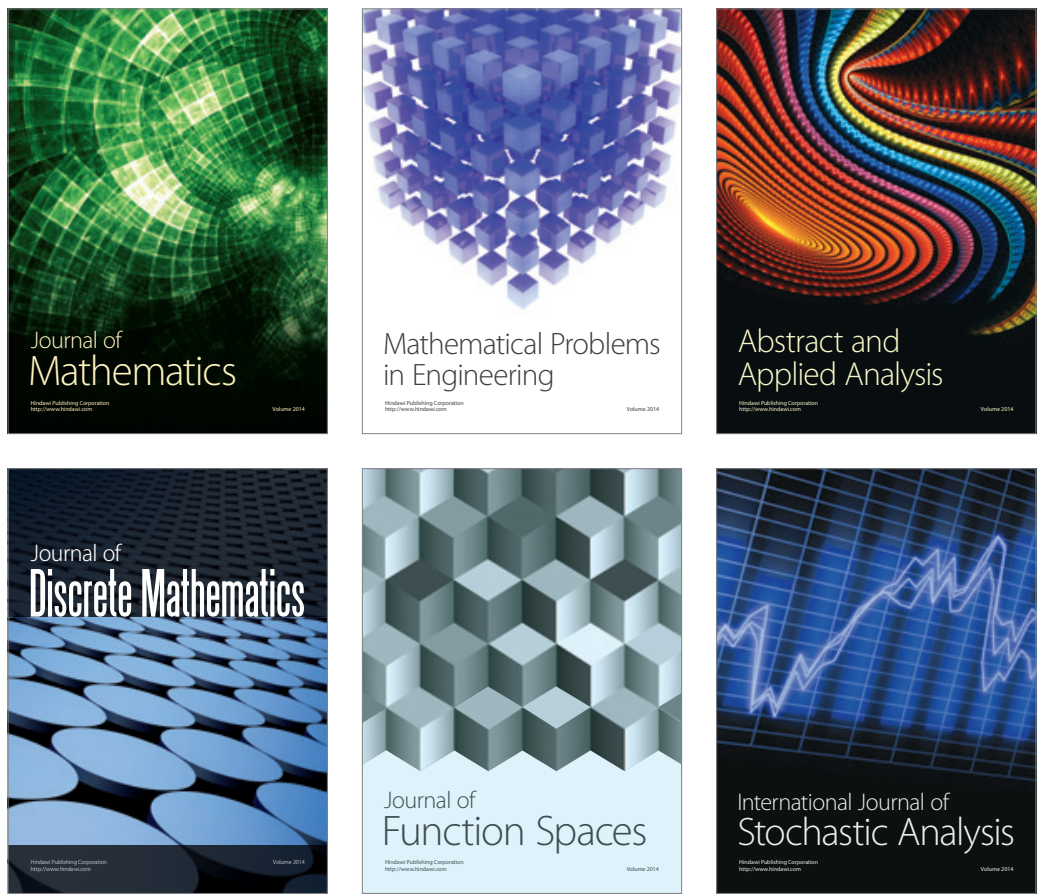

Journal of

Function Spaces

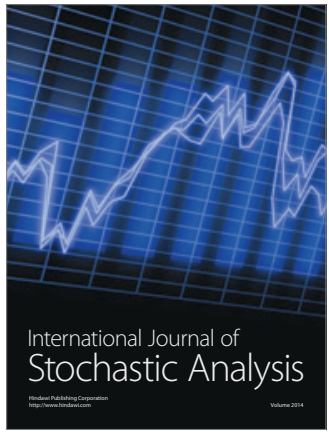

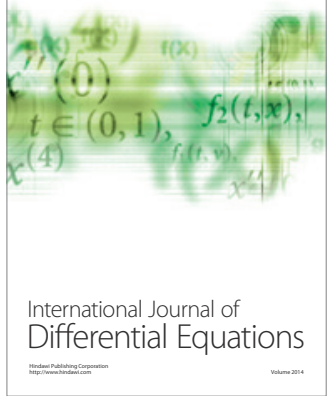
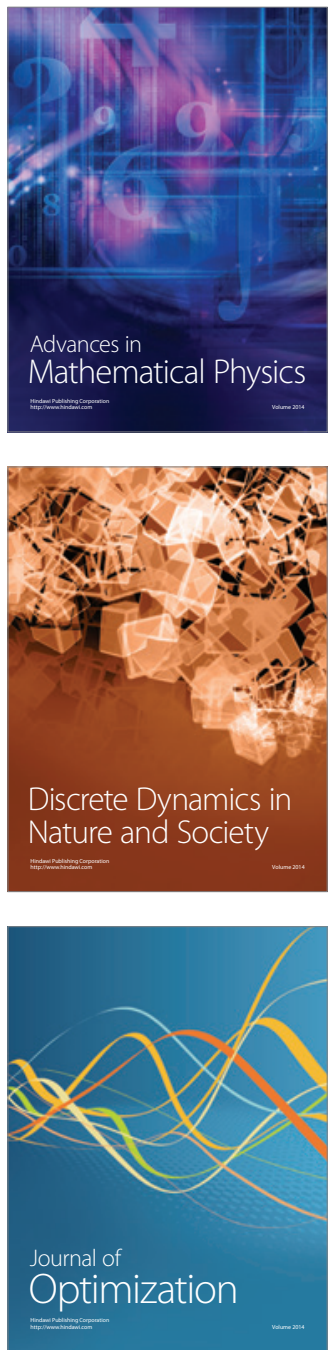